\title{
Diseño de la plataforma RedacText 2.0 para ayudar a escribir textos académicos e investigar sobre enseñanza y aprendizaje de la escritura
}

\section{The design of the digital platform RedacText 2.0 to aid writing and researching academic texts}

\author{
Teodoro ÁlVAREZ ANGULO ${ }^{1}$, Teresa MATEO GIRONA ${ }^{1}, \mathrm{M}^{\mathrm{a}}$ del Pilar SERRANO \\ ALMODÓVAR $^{1}$ y Miguel Ángel GONZÁLEZ SERRANO ${ }^{2}$ \\ ${ }^{1}$ Universidad Complutense de Madrid y ${ }^{2}$ VASS
}

Recibido: Noviembre 2013

Aceptado: Abril 2014

\begin{abstract}
Resumen
Este trabajo da cuenta del diseño experimental de una plataforma interactiva (RedacText 2.0), concebida para ayudar a redactar textos académicos a estudiantes universitarios, futuros maestros de Educación Primaria y futuros profesores de Educación Secundaria. Los principios que sustentan el diseño de esta herramienta están en la línea de programas anglosajones, como: Writing Across the Curriculum, Writing in the Disciplines, Expository Writing, Writing Program, Reading and Writing to Learn, Research on Writing; y movimientos del tipo de: European Association for the Teaching of Academic Writing, Scaffolded Writing and Rewriting in the Disciplines, Center for Academic Writing, y Nacional Writing Projet, entre otros. Este recurso aborda el contexto, el proceso de producción y el texto (expositivo o académico, para el caso), con ayudas que facilitan la realización de las tareas de composición, tanto por parte del estudiante o escritor como del profesor o tutor. Esta plataforma consta de tres secciones: tutor, escritor y Grupo Didactext. La sección dedicada al estudiante o escritor permite descargar actividades, que se envían electrónicamente, con el fin de que el tutor guíe y evalúe el desarrollo de la producción del texto y medie en los aprendizajes. Esta sección permite crear una base de datos que registra los progresos en los aprendizajes de cada escritor y contribuye a mejorar sus destrezas como escritor. La documentación generada en esta sección permite al Grupo Didactext investigar acerca de cómo enseñar y aprender a escribir trabajos académicos, desde la perspectiva que provocan en los lectores los procesos de lectura y de escritura con la ayuda de Internet. Este trabajo tiene como ámbito de aplicación principal la formación del profesorado.
\end{abstract}

Palabras clave: plataforma interactiva, autoaprendizaje, autoevaluación, investigación en escritura académica, formación del profesorado.

\footnotetext{
Abstract

This paper deals with the analysis, design and implementation of an interactive platform (RedacText 2.0) aimed to aid college students and future teachers of elementary and secondary education write academic texts. As a resource, RedacText 2.0 addresses the context and the 
production process of academic texts. It is equipped with help tools which facilitate the completion of tasks, both by the writer and the tutor. This platform consists of three sections: Tutor, Writer and Didactext group research, and focuses on context, production process and text. The writer section includes activities available for download and allows them to be sent electronically to the tutor, so that he can guide and evaluate the process of the production of the text and mediate in the writer's learning. This section allows students to create a database which records progress in their learning and contributes to improve their writing skills. The documents generated in this section also allow Didactext research group to investigate how to teach and learn academic writing, and pays particular attention to online sources/resources. The main goal of this project is to contribute to our understanding of teacher training. The project is in line with other writing programs in English such as: Expository Writing, Writing Programs, Center for Academic Writing; and trends similar to: European Association for the Teaching of Academic Writing, Scaffolded Writing and Rewriting in the Disciplines; and the National Writing Project.

Keywords: interactive platform, autonomous-learning, self-assessment, research on teaching and learning academic writing, teacher training.

Varias son las razones que justifican el diseño y la aplicación de la plataforma RedacText 2.0 para ayudar a escribir textos académicos; entre las principales, destacan las siguientes: (i) la escritura es una herramienta social poderosa en cuanto que capacita para adquirir conocimiento, construir pensamiento y comunicarse con los demás; (ii) la comprensión y la expresión escritas son ejes fundamentales de la cultura; (iii) escribir, más que sólo leer, proporciona voz a la gente, $\mathrm{y}$, por tanto, poder, ya que la escritura se asocia principalmente con participación y desavenencia; (iv) en el ámbito académico, la escritura es una herramienta con finalidades como: aclaración de conceptos o ideas, resolución de problemas, demostración y transmisión de información, transformación del conocimiento, y recurso para vivir en comunidad; (v) la informática ha transformado la escritura. La composición digital se concibe como escritura para compartir, dialogar, y participar; (vi) este recurso facilita la autonomía en el aprendizaje, mediante el acceso a documentación y a herramientas de ayuda; propicia la construcción del conocimiento; y fomenta la evaluación y la autoevaluación del proceso de aprendizaje; y (vii) en la formación del profesorado, es un recurso fácilmente replicable por los usuarios en sus aulas futuras.

Todo ello supone considerar la escritura como un fenómeno complejo, que requiere atención especial en contextos de formación del profesorado, tanto en su formación académica como en su formación profesional.

\section{¿Qué se pretende con la plataforma RedacText?}

El diseño de la plataforma RedacText 2.0 persigue dos objetivos principales: (a) motivar y tutelar a futuros maestros de Primaria y a futuros profesores de Secundaria, en la producción de textos académicos, con el fin de favorecer su competencia discursiva escrita; (b) ofrecerles, a lo largo de su formación profesional inicial, recursos de enseñanza que puedan ser descargados on line. Se trata, por tanto, de la presentación del proceso de producción de textos académicos o expositivos, basado en 
los principios de la Web 2.0, y de las correspondientes actividades, estrategias y modelos que faciliten la composición de textos académicos.

La formación de estos estudiantes universitarios pretende asimismo capacitarlos didácticamente, para que puedan enseñar a sus futuros alumnos e interesarse por cómo aprenden a escribir en las diferentes áreas del currículo que prescribe la normativa oficial (LOE 2/2006, de 3 de mayo y el desarrollo normativo de la correspondiente Comunidad Autónoma). A tal efecto, el diseño de esta plataforma contempla la posibilidad de que los futuros maestros y profesores puedan replicar este recurso para ayudar a sus alumnos.

\section{¿Cómo está diseñada la plataforma?}

El procedimiento seguido en el diseño de la plataforma es el siguiente:

i. Construcción del marco teórico que fundamenta el proyecto (secuencia didáctica, proceso de escritura y regularidades lingüísticas del texto).

ii. Elección de los dos grupos de experimentación, como se indica más adelante.

iii. Elaboración de materiales didácticos y herramientas para el desarrollo de cada fase del proceso de escritura: artículos y presentaciones power point para el profesor; artículos con diversas ayudas (desplegables y enlaces) y cuestionarios descargables para los estudiantes. Estos cuestionarios sirven para dejar constancia de cómo se progresa, antes de pasar a la siguiente fase del proceso de escritura.

iv. Experimentación de los materiales diseñados con los dos grupos de estudiantes, desarrollada en tres momentos: a) antes: presentación teórica de la fase; b) durante: realización de la plantilla, puesta en común de dificultades y mejoras, y entrega de los productos obtenidos; c) después: análisis, valoración de los resultados, y modificación de los materiales por el grupo de investigadores.

v. Organización de los materiales en la plataforma, a cargo del equipo de investigación y del equipo de informáticos, de este modo: a) análisis y reflexión de la distribución de los contenidos y del diseño de los mismos en la plataforma; b) primera versión de la plataforma, de acuerdo con lo consensuado en la etapa anterior; c) pruebas y validación de la versión definitiva de RedacText 2.0.

La plataforma consta de: área del profesor, área del estudiante y área del Grupo Didactext. El área del profesor contiene un artículo y un power point mediante los que se explica en qué consiste y cómo se lleva a cabo cada fase del proceso (acceso al conocimiento, planificación, producción, y revisión y reescritura). El área del estudiante contiene un artículo explicativo y una plantilla interactiva, con ayuda de desplegables, referidos al contexto, a la estructura y a las regularidades lingüísticas y textuales de los textos expositivos o académicos..

Los materiales didácticos son ayudas para producir textos que promuevan la transmisión de información y la transformación de conocimientos, por un lado; y para 
resolver problemas retóricos relacionados con la producción de textos académicos (concepción epistémica de la escritura), por otro. Además, se ha creado un banco de recursos online, que contribuye a mejorar la escritura académica, y a utilizar de manera eficaz las tecnologías de la información y de la comunicación para la enseñanza. Todo ello favorece el aprendizaje y la evaluación autónomos.

El desarrollo técnico de la plataforma se lleva a cabo de la siguiente manera: el equipo de investigación y el de informáticos analizan, en sesiones semanales, cómo diseñar la plataforma, cómo organizar los contenidos, cómo gestionar la zona estática y la zona interactiva; qué tipo de contenidos teóricos debe incluir, qué tipo de materiales didácticos, etc. El equipo técnico presenta una primera versión de la plataforma, de acuerdo con lo consensuado en cada fase. Finalmente, se corrigen los materiales, se revisan y se prueba el área interactiva, hasta llegar a la versión definitiva de la plataforma.

\section{¿Quiénes son los usuarios de esta plataforma?}

La validación del procedimiento de producción de textos se lleva a cabo con dos grupos de estudiantes universitarios. Uno, en la asignatura "Didáctica de la Lengua Española", correspondiente al tercer curso del Grado en Maestro de Educación Primaria (GMEP); el otro, en la asignatura "Innovación e Investigación en Lengua Castellana y Literatura", perteneciente al Máster en Formación del Profesorado de Secundaria (MFPS). Cada grupo consta de seis miembros. Ambos grupos de estudiantes tienen como característica común que se forman para el ejercicio profesional en sendos tramos de la Educación Obligatoria.

Los usuarios de esta plataforma aprenderán: (i) en qué consiste el proceso de escritura (fases y actividades); (ii) cómo llevarlo a cabo (modelos de escritura y material didáctico que podrá ser descargado); (iii) cómo beneficia a los usuarios (adquisición significativa de conocimientos, perfeccionamiento de la competencia discursiva); (iv) cómo realizar la autoevaluación del proceso de escritura; y (v) cómo guiar este proceso en el aula.

Esta plataforma es replicable asimismo por usuarios distintos de los descritos anteriormente, tales como maestros de Primaria y profesores de Secundaria en ejercicio, así como en diferentes ramas de la Formación Profesional, educación a distancia, y educación de adultos, entre otros.

\section{Supuestos en que se basa el diseño el marco teórico}

Los presupuestos teóricos que sustentan nuestro trabajo son los siguientes:

i. Escribir es una habilidad lingüística compleja; por lo que es necesario explicitar el proceso con el fin de que el que escribe sea consciente de la complejidad de esta habilidad, lo interiorice, mediante la actividad, y lo llegue a automatizar, y así sea competente en los diferentes géneros discursivos que exige el ámbito académico, profesional y social. 
La reflexión acerca de la composición escrita ha generado diversos modelos que enfatizan, ya sea sus productos, ya sea el proceso, o la forma de aprender y enseñar a escribir. El modelo Didactext (2003) que inspira el diseño de la plataforma se basa entre otros de los modelos de Hayes \& Flower, 1980; Hayes, 1996; Bereiter \& Scardamalia, 1987; Vigotsky, 1987; Gregg \& Steinberg, 1980; Nystrand, 1982; Hamilton \& Barton, 1985; Olson, 1994; Candlin \& Hyland, 1999; Grabe \& Kaplan, 1996; Dijk, 1997a y 1997b.

ii. El conocimiento de las regularidades lingüísticas y textuales de los géneros de exposición de información que requiere la escritura en el ámbito académico y profesional propicia una mejor comprensión y producción de tales textos. Esto es lo que podríamos denominar "gramática de la exposición" (De Beaugrande \& Dressler, 1972/1997; Halliday, 1982; Halliday \& Martin, 1993; Britton \& Black, 1985; Bernárdez, 1987; Combettes, 1988, 1992; Adam, 1992; Bronckart, 1996; Álvarez, 2001, 2005 y 2010).

iii. El trabajo con secuencias didácticas centradas en temas de interés para el que escribe y las exigencias de los distintos géneros propios de las materias del currículo, favorecen los productos de escritura académica. Esto es lo que postulan movimientos que estudian la escritura, como: Academic Writing, Writing Across the Currículo, Writing in Disciplines, Writing to Learn, Scaffolding Academic Literacy; y autores como: Graves, 1983; Wray \& Lewis, 1997; Bazerman, 2005; Reuter, 1996; Halté, Petitjean y Plane, 2002; Dolz, Noverraz \& Schneuwly, 2001; Schneuwly, B., 1988; Dolz, Gagnon \& Toulou, 2008; Simard, Dufays, Dolz, \& García-Debanc, 2010; Castelló, 2007; Castelló y Donahue, 2012; Carlino, 2006; Camargo, Uribe y Caro, 2011; Ramírez, 2010; Didactext, 2006a, 2006b, 2007; Camps, 2003; Sanmartí, 1996 y 2007; Álvarez Angulo, 2006, 2007 y 2011; García, 2011; Álvarez y García, 2011; Rose \& Martin, 2012.

iv. Internet, con herramientas como la Web 2.0, provoca cambios en la didáctica de los procesos lectores y escritores, permite el paso de una enseñanza pasiva a una colaborativa y cooperativa, ya que pone al alcance del profesorado y del alumnado nuevos recursos didácticos, como Wikis (Olive \& Levy, 2002). La Web 2.0 también facilita la consulta, creación y edición de contenidos, además de poder decidir cómo, cuándo y dónde los consulta, crea y edita. Dicho en otros términos, RedacText 2.0 facilita el multi-acceso a la información, promueve la autonomía y la autoevaluación de los aprendizajes e integra herramientas útiles y prácticas que guían el proceso de escritura dentro y fuera del aula. La plataforma está dotada de una integración directa con Twitter, de tal forma que muestra un historial con todas las publicaciones que se han lanzado a la herramienta social de microbloging Twiter (Java, Song, Finin \& Tseng, 2007; Honey \& Herring, 2009). Asimismo, esta plataforma está integrada con las principales redes sociales (Wasserman, 1994; Ellison, 2007), Meneame, Facebook y Twitter, lo que facilita la colaboración y la distribución de los contenidos entre diversos tipos de usuarios.

v. Desde supuestos de investigación didáctica, interesa la observación y el análisis del uso que hacen de la herramienta docentes y alumnos, y la relación que se 
establece entre el marco epistemológico, los currículos, las materias de enseñanza, así como la tarea de mediación, y las ayudas que llevan a cabo los docentes en las aulas.

\section{Metodología}

Instrumentos

Los instrumentos que se han utilizado para el diseño han sido los siguientes:

- Secuencia didáctica basada en el modelo Didactext (Grupo Didactext, 2006b).

- Artículos y presentaciones power point para el profesor, en cada fase del proceso de escritura.

- Artículos con diversas ayudas (desplegables y enlaces) para guiar a los estudiantes.

- Plantillas o cuestionarios que pueden descargarse y que ayudarán a los estudiantes a reflexionar y a tomar decisiones respecto del contenido y de las características del texto. Estas herramientas servirán, además, para dejar constancia de cómo se progresa antes de pasar a la fase siguiente.

\section{Procedimiento}

El desarrollo del proyecto se ha llevado a cabo de la siguiente manera:

\section{Fase preparatoria}

En un primer momento, se establece el calendario de reuniones del grupo de investigación para la realización de las tareas siguientes:

- Fundamentación teórica. Construcción del marco que sustente el proyecto.

- Recopilación y análisis de la documentación correspondiente al marco legal de Educación Obligatoria.

- Planificación de la puesta en práctica del proyecto. Los acuerdos adoptados son los siguientes:

- Diseño de una fase experimental cuyo objetivo general sea producir un texto expositivo de divulgación, siguiendo las fases del proceso de escritura, sobre un tema de libre elección, relacionado con la asignatura Conocimiento del Medio (en Educación Primaria) o de Ciencias de la Naturaleza (en Educación Secundaria), de unas 2000 palabras. El texto debe ser producido de manera individual.

- Elección de dos grupos de experimentación: GMEP y MFPS.

- Preparación de la toma de contacto y de las sesiones informativas con los estudiantes.

- Calendario de las sesiones de trabajo del grupo de investigación y de los grupos de estudiantes con los investigadores. 
- Designación de los profesores encargados de dinamizar las diferentes sesiones, así como de un relator de la dinámica de trabajo de ambos grupos.

- Iniciación del contacto con el equipo informático encargado de diseñar y programar los trabajos de la plataforma.

\section{Fase de diseño del material didáctico}

El grupo de investigación se divide en dos subgrupos de trabajo (GMEP y MFPS), encargados de diseñar el material didáctico de cada fase del proceso de escritura. Ambos subgrupos elaboran una propuesta didáctica para orientar a los estudiantes. En las sesiones de trabajo del grupo de investigación se analizan, corrigen y reformulan los cuestionarios, con las actividades correspondientes a cada fase, y se les da el visto bueno para su puesta en práctica.

Junto con la elaboración del material didáctico, los integrantes de cada subgrupo preparan artículos teóricos que para cada fase, destinados al área del profesor. Para el área del estudiante, los textos son más sencillos, prácticos y divulgativos. Unos y otros textos son analizados y reformulados, a su vez, por el conjunto de los investigadores, hasta que se estiman adecuados para su incorporación en la plataforma.

\section{Fase de validación}

La puesta en práctica del material didáctico tiene como objetivo validar la versión preliminar de lo que posteriormente será el área interactiva de la plataforma. El propósito de esta fase del proyecto consiste en que los participantes produzcan el texto expositivo anteriormente mencionado.

Ambos grupos participan en un total de siete sesiones, a lo largo de las cuales se presenta el proyecto, se llevan a cabo las diferentes etapas del proceso de escritura y se debate la experiencia en sus diversas dimensiones.

Las sesiones se celebran semanalmente y tienen como objetivos principales:

- Suministrar a los participantes en la investigación la versión impresa del material didáctico que les va a guiar a través de cada fase del proceso de escritura.

- Debatir sobre los aspectos positivos y negativos del material didáctico de la fase anterior -utilidad, posibles modificaciones, propuesta de materiales y herramientas para facilitar el proceso de escritura, etc.

- Analizar las dificultades encontradas y los logros alcanzados.

- Entregar los productos de las diferentes fases (por ejemplo, con respecto a la fase de acceso al conocimiento: notas, lluvia de ideas...; en lo referente a la fase de planificación: esquemas, resúmenes, guiones o índices; en la fase de producción: el primer borrador; en la fase de revisión y reescritura: las pautas de revisión, y, al final, el texto definitivo). 
En cada sesión, y en ambos grupos, se comenta el proceso y los resultados de la fase ya realizada, y se introduce la fase siguiente. El relator toma nota y registra los aspectos relevantes de las sesiones, grabadas en audio y posteriormente transcritas.

A continuación, se describe, a modo de ejemplo, el procedimiento llevado a cabo con los grupos de validación, mediante la trascripción de las reflexiones y los comentarios que hacen.

\section{a) Primera Sesión:}

1. Introducción y presentación del proyecto (power point de presentación). Los investigadores explican los contenidos teóricos relacionados con la primera fase. Comienzan preguntando a los estudiantes para ver cuáles son sus conocimientos previos y se explica a partir de los mismos.

2. Entrega y explicación del documento de actividades. Los estudiantes del GMEP muestran sus inquietudes. La más destacable es que se sienten inseguros en el proceso de escritura. Piden textos que les sirvan de modelo. Estas consideraciones son tenidas en cuenta por el grupo de investigación.

3. Introducción a la fase de acceso al conocimiento. Los estudiantes formulan preguntas y hacen comentarios. Destaca, entre los estudiantes del GMEP, la preocupación por la elección del tema.

b) Segunda Sesión:

4. Comentario sobre las sensaciones, impresiones, dificultades, etc., con ayuda de preguntas, respecto a la fase de acceso al conocimiento. Esto dice un estudiante del MFPS: "Me ha resultado constructiva la plantilla porque tiendo a divagar y a usar muchas subordinadas. Y esto me ha ayudado a concretar. Para los dispersos ayuda muchísimo".

El profesor-investigador inicia una ronda con la siguiente pregunta: "¿Qué elemento os ha ayudado a determinar, a concretar el tema?". Las respuestas de los alumnos del grupo MFPS se resumen en lo siguiente: el elemento que más ayuda es pensar en el destinatario, en el género discursivo, en el emisor (sus propias limitaciones), en el tema (saber que tienes que escribir 2000 palabras y poder tener información suficiente).

5. Los estudiantes aportan ideas, proponen modificaciones, etc., para la plataforma.

Una estudiante del MFPS comenta: "Para mí es un inconveniente separar esas dos etapas (acceso al conocimiento y planificación). Yo no puedo imaginar hacerlo de otra manera. No podría haberlo hecho en dos fases, y lo he hecho en una sola".

6. Los estudiantes aportan los productos de la fase de acceso al conocimiento. Se ciñen a cuestiones como: ¿De qué quiero hablar?, ¿qué necesito saber?, ¿qué sé del texto expositivo?, entre otras. 
c) Tercera Sesión:

7. Introducción a la fase de planificación.

8. Comentario sobre las concepciones, sensaciones, impresiones, dificultades, etc., con ayuda de preguntas que realizan los investigadores, y que recoge por escrito el relator.

Los estudiantes comentan que les es útil replantearse a quién va dirigido el texto (destinatario o audiencia), lo que les hace ser conscientes de las modificaciones que tienen que introducir.

9. Los estudiantes aportan ideas y proponen modificaciones para incluir en la plataforma.

d) Cuarta Sesión:

10. Los estudiantes aportan los productos de la fase de planificación. Como ejemplo, una estudiante del GMEP presenta un mapa conceptual sobre el tema, y un estudiante del MFPS, un esquema-guión con los siguientes apartados: introducción, la tectónica de placas y las erupciones fisurales: Canarias.

11. Introducción de la fase de producción textual.

12. Comentario sobre las concepciones, sensaciones, impresiones, dificultades, etc., de los dos grupos, con ayuda de preguntas realizadas por los investigadores, respecto de la fase de producción textual.

Los estudiantes destacan la utilidad de los siguientes consejos: evitar las oraciones subordinadas, construir párrafos y usar conectores lógicos para unir párrafos. También comentan que son teorías conocidas pero que no se recuerdan a la hora de escribir, por lo que les resulta útil tenerlas presentes en el momento de la escritura para hacer uso de ellas.

Entre las dificultades que se encontraron, merecen destacarse las relacionadas con los siguientes aspectos:

- La extensión requerida. Un estudiante comenta que ha tenido que suprimir la segunda parte de su texto.

- Los subtipos. La elección del subtipo, en la primera fase, les ha parecido un esquema rígido. Los profesores insisten en que pueden ser varios y que su elección puede variar a lo largo del proceso, ya que la escritura es un proceso recursivo; por tanto, pueden mezclar subtipos aunque siempre suele haber uno dominante. Esta cuestión se reformulará para que quede claro en la plataforma.

- Las consultas gramaticales: piden que se facilite una web con este recurso.

- La construcción de párrafos.

- La subordinación.

- El ajuste de la información y del lenguaje al grado de conocimientos del tema elegido. 
e) Quinta Sesión:

13. Los estudiantes de ambos grupos aportan ideas, proponen modificaciones, etc. El relator toma nota de cada una de ellas, y el grupo de investigación introduce en la plataforma las modificaciones consensuadas.

14. Los estudiantes aportan los productos de la fase de producción textual, relacionados con los subtipos de textos explicativos que se van a utilizar (la clasificación y la definición-descripción) y con los principales mecanismos de cohesión textual.

15. Se introduce la fase de revisión y reescritura.

f) Sexta Sesión:

16. Comentario sobre las concepciones, sensaciones, impresiones, dificultades, etc., con ayuda de preguntas, respecto de la fase de revisión.

Comparten la misma concepción de la revisión en ambos grupos: antes sólo corregían cuestiones de ortografía; ahora, gracias a la plantilla entregada, se han dado cuenta de que siempre se puede cambiar y mejorar lo que se ha escrito. Les ha servido para reorganizar epígrafes, modificar el título y la estructura, revisar cuestiones de estilo, usar conectores, emplear sinónimos, adecuar el registro al destinatario, replantearse el uso de imágenes y gráficos.

17. Los estudiantes de ambos grupos aportan ideas, proponen modificaciones, etc., para la plataforma.

Piden que se proporcione información sobre cómo introducir gráficos (fotos, tablas, etc.). Además, sugieren que ciertas preguntas se reformulen aclarando que no siempre deben emplearse los recursos que se aconsejan. Por ejemplo: el uso de marcas de impersonalidad. No se trata de que todo se escriba en impersonal, sino que es una característica de estos textos.

18. Los estudiantes aportan los productos de la fase de revisión y reescritura.

g) Séptima Sesión:

19. Valoración global del proyecto: logros y propuestas de mejora. A continuación, transcribimos algunos comentarios realizados por los estudiantes:

E1: Le ha servido para acotar (algo que le cuesta muchísimo). La planificación y la fase de acceso al conocimiento le han resultado de mayor ayuda que la de redacción. Respecto a la autocorrección, apunta que muchas veces leemos lo que queremos leer; por tanto, es difícil autocorregirse.

E2: Detecta que no se enseñan suficientemente en la escuela las fases de planificación y revisión, que son las que le han parecido más útiles. 
E3: Como a E2, las fases que más le han ayudado han sido la de planificación y la de revisión. Respecto a la fase de acceso al conocimiento, procede así de forma automática en la escritura académica.

E4: Describe el proceso como una experiencia muy enriquecedora y considera que este tipo de herramientas es muy útil para enseñar textos expositivos. Las fases de acceso al conocimiento y de planificación las tiene ya muy interiorizadas. Tal vez lo que le haya aportado más como escritora es la revisión, que, además, nunca se enseña.

E5: Detecta que nunca habíamos reflexionado tanto en el proceso de creación de un texto. Como E1, piensa que muchas veces, en la corrección, no vemos nuestras propias erratas y apunta que se podría trabajar la corrección colaborativa. Le ha ayudado la idea de pensar en la pregunta-guía a la que se quiere responder a lo largo del texto.

\section{Fase de reformulación}

Una vez concluida la fase de validación, el grupo de investigación propone mejoras, tanto de los artículos teóricos, como de los cuestionarios y enlaces de ayuda, en cada fase del proceso de escritura. Para la introducción de los cambios en los materiales y herramientas se tienen en cuenta las propuestas de los estudiantes. En este sentido, la labor de la relatora es fundamental porque ofrece información respecto de cómo han resuelto las actividades los estudiantes de ambos grupos, comparando y analizando las dificultades encontradas por unos y otros. Asimismo, son de gran interés los comentarios sobre la utilidad de los materiales entregados y sobre el material adicional que se considera necesario aportar.

Ambos grupos proponen adaptaciones del material, en cada fase del proyecto; tras ser consensuadas con el equipo investigador, se le entregan al técnico para su inclusión en la plataforma.

A continuación, se muestra una relación de las aportaciones de los dos grupos de estudiantes a las distintas fases, conforme puede verse en la tabla 1.

\section{Fase I}

- Añadir textos que sirvan de modelo.

- Suprimir la elección de subtipos de textos expositivos en esta fase, pues está más relacionada con la planificación que con la búsqueda de información.

- Reformular la pregunta: “QQué necesito saber?”.

- Añadir instrucciones sobre cómo se debe buscar la información.

- Insistir en la idea de tomar nota de la bibliografía consultada.

- Aclarar qué producto se espera que se realice al hacer la búsqueda de información para que los estudiantes no se adelanten a la fase siguiente, y no organicen la información mediante esquemas o índices.

- Explicar en qué consiste la contextualización y aportar ejemplos.

- Añadir géneros relacionados con el contexto escolar.

- Incluir una ayuda con información sobre el texto expositivo. 
- Reformular la instrucción "uso de la $3^{\mathrm{a}}$ persona en los textos expositivos" por: "Predominio de las formas no personales o impersonales".

- Explicar en qué consisten los subtipos definición y descripción.

- Añadir ejemplos de subtipos del texto expositivo.

\section{Fase II}

- Cambiar el epígrafe "Antes de redactar": crea confusión.

- Añadir destinatarios relacionados con el ámbito educativo.

- Dar instrucciones claras sobre cómo hacer el desarrollo para que no se adelanten a la siguiente fase.

- Precisar el producto que se espera con los siguientes términos: esquema, mapa conceptual o índice.

\section{Fase III}

- Aclarar qué significa "adecua el texto al registro", mediante un ejemplo en el que se vea que el registro es adecuado, y otro, en el que no lo es.

- Especificar que para saber si "las ideas están secuenciadas en orden jerárquico" pueden revisar el esquema de la fase de planificación.

- Aclarar qué significa "busca el equilibrio entre la información nueva y la conocida" con la explicación de los conceptos de tema, rema y progresión temática. Se confunde el término "conocida", relacionándolo con conocimientos previos.

- Reducir la lista de errores sintácticos y morfosintácticos poniendo sólo los más comunes.

- Añadir ejemplos en la lista de errores sintácticos y morfosintácticos, especialmente en la explicación de leísmo, laísmo y loísmo.

- Eliminar la pregunta sobre el equilibrio de los párrafos.

- Explicar cómo se introducen los elementos paratextuales.

- Presentar el uso de cita como un recurso textual optativo, en vez de como un recurso necesario.

- Añadir explicaciones para realizar referencias bibliográficas.

- Presentar las ayudas textuales de carácter optativo, a continuación del formulario para hacer el borrador y como desplegables que se abrirán cuando el estudiante quiera obtener más información sobre cada ítem.

\section{Fase IV}

- Añadir enlace a páginas web con correctores ortográficos y sintácticos.

- Sólo añadir información nueva acerca de los aspectos que se explican por primera vez en la plantilla. Las cuestiones explicadas en las fases anteriores se presentarán como desplegables.

- Simplificar las opciones de respuesta para cada pregunta del cuestionario. En caso negativo, en lugar de introducir otras dos opciones, dar la instrucción concreta para reescribir ese aspecto de su texto.

- En la pregunta “¿repites muchas veces una palabra?”, añadir “siempre que sea posible” para relativizar la necesidad de utilizar palabras distintas siempre.

Tabla1. Aportaciones de los estudiantes a las distintas fases. 


\section{Utilización de esta plataforma}

Esta plataforma ha sido desarrollada siguiendo la metodología COBAS-M (Corporative Business Architecture Software Method), conforme a la propuesta que está siendo desarrollada en la tesis doctoral de uno de los autores del trabajo. Se Compone de diferentes secciones, como se puede ver en la figura 1:

- Menú Principal: da acceso a las dos áreas que representan a los principales usuarios del sistema: profesores y estudiantes. En un futuro próximo, se podrá ampliar el espectro de usuarios.

- Menú Secundario: permite el acceso individual a cada una de las fases del proceso de escritura, desde dos puntos de vista: el de profesor y el del estudiante. Asimismo, este elemento técnico de la plataforma no limita a un acceso secuencial sobre las fases del proceso, sino que permite acceder a la información de cada fase sin importar el orden. También ofrece un acceso a información general sobre el Grupo Didactext.

- Área principal: muestra los artículos que explican las diferentes fases del proceso, así como la actividad interactiva propuesta en cada sección, y ofrece una interacción entre el usuario y la plataforma. Esta área incluye información en diferentes soportes electrónicos (integración con SlideShare para mostrar presentaciones en formato Microsoft Power Point). También aporta desplegables desarrollados con tecnología JavaScript y AJAX, para la visualización de las ayudas contextuales que pueda necesitar el usuario.

- Integración con Redes Sociales: desde este elemento del portal se permite la publicación de contenidos hacia las principales tecnologías de la Web 2.0 (Facebook, Twitter, etc).

- Integración con Twitter: gracias a esta sección de la plataforma, los miembros del proyecto pueden referenciar mensajes en Twitter, publicados de forma automática en esta sección, con el fin de crear un índice de contenidos interactivo y online. 


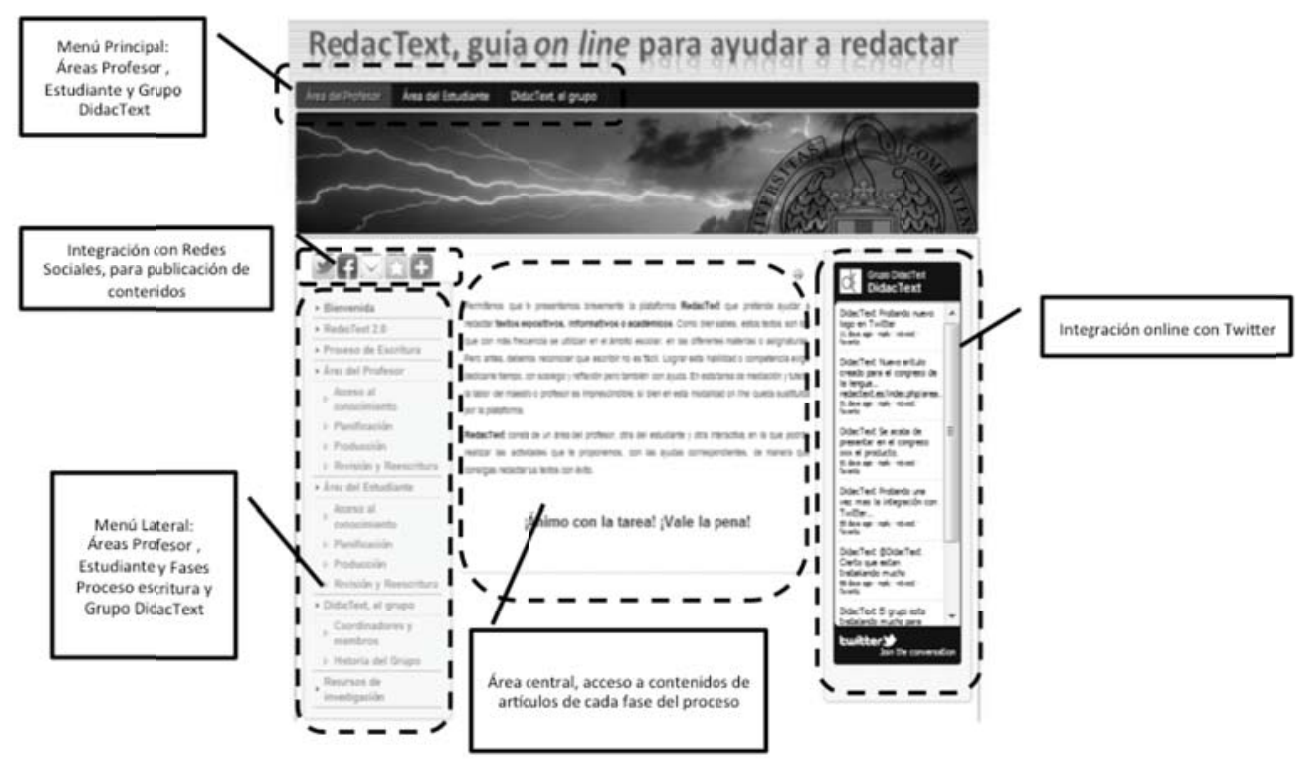

Figura 1: Secciones de la plataforma RedacText 2.0.

La plataforma RedacText 2.0, según se ilustra en la figura 2, ofrece diferentes vías de interacción para sus usuarios; a saber:

- Estudiante: acceso al contenido de información sobre las fases del proceso y sobre las actividades interactivas. El resultado de cada actividad produce un envío de información electrónica al correo del estudiante, y posibilita la creación de una base de datos de sus resultados.

- Profesor: acceso al contenido de información de las fases del proceso.

- Proyecto: automáticamente la plataforma envía los resultados de todas las actividades realizadas por todos los usuarios (perfil estudiante), y crea una fuente de información que permite la explotación y el análisis posterior de estos datos para la elaboración de estadísticas con métricas e indicadores.

- Usuarios de proyecto y externos: gracias a la integración con Twitter, los usuarios de RedacText podrán publicar mensajes en el historial de Twitter. 


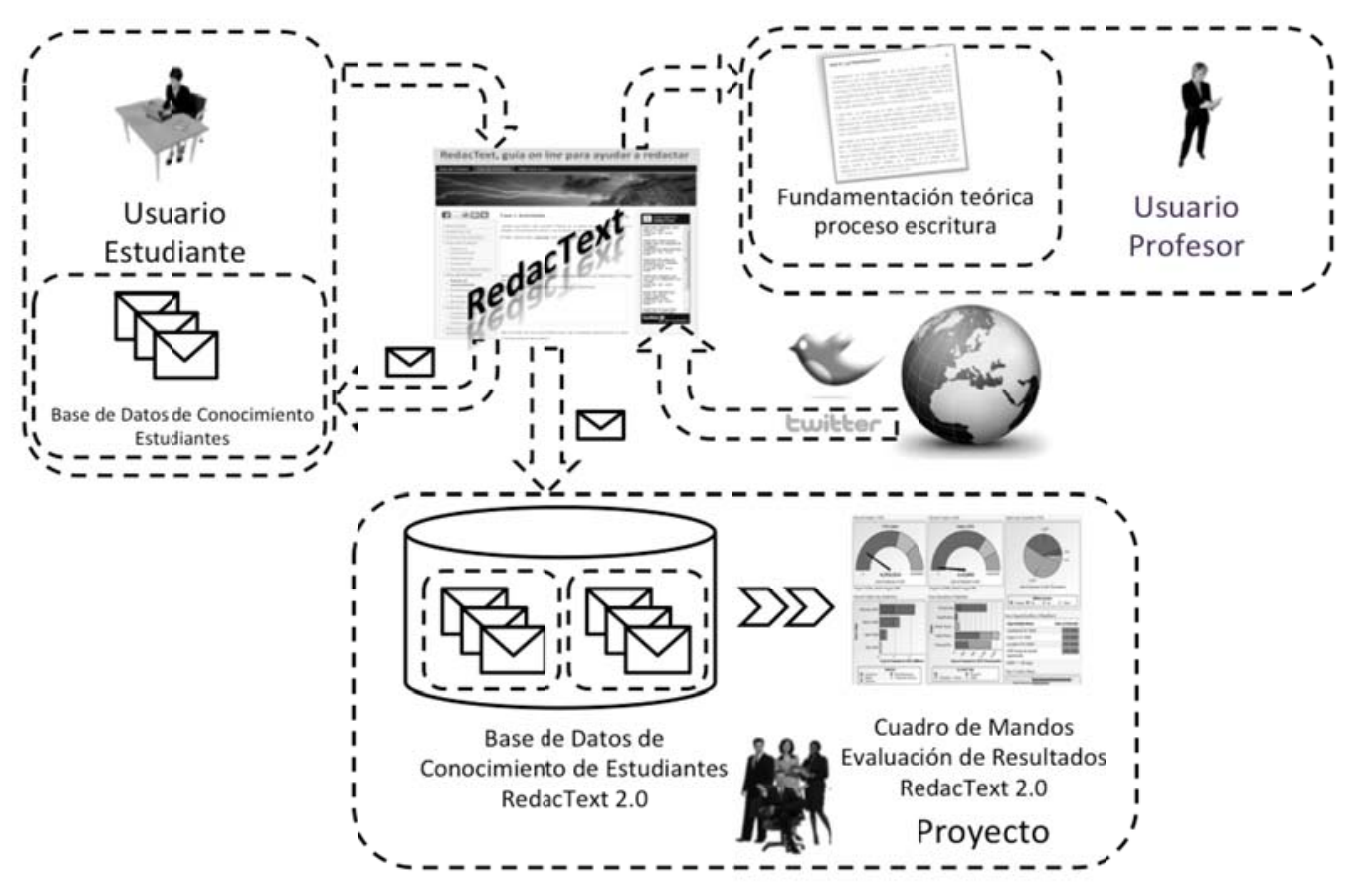

Figura 2: Modelo de interacción de los usuarios de RedacText.

\section{Ventajas y proyección de futuro de RedacText 2.0}

Las ventajas y la proyección de futuro de RedacText 2.0 son las siguientes:

a) Respecto de los estudiantes. Esta plataforma favorece el aprendizaje autónomo, de forma guiada y asistida electrónicamente, así como la creación de una base de datos de conocimiento para evaluación de actividades de los estudiantes, etc.

b) Respecto de los profesores. Este recurso propicia: (i) la fundamentación teórica y la utilización de actividades y materiales en las aulas; (ii) la aportación de comentarios de evaluación sobre las actividades realizadas por los estudiantes en la plataforma; y (iii) la posibilidad de consulta de resultados al histórico de estudiantes.

c) Respecto del Grupo de Investigación Didactext. Esta herramienta permite:

- La creación de una base de datos mediante el envío de información electrónica con la información de todos los usuarios, en la que se puede intervenir para mejorar los resultados.

- La segmentación de usuarios mediante el análisis de actividades de estudiantes/profesores, según variables y tipos de usuarios.

- La evaluación de estudiantes, en la que se tenga en cuenta la temporalidad y el momento del periodo de aprendizaje. 
- La elaboración de métricas e indicadores sobre las actividades realizadas en el portal.

- La gestión de contenidos: se trata de una plataforma interactiva que permite la aportación de nuevos contenidos de fácil actualización.

- La integración con Web 2.0: RedacText, como plataforma online, está integrada con las principales redes sociales para favorecer la distribución de contenidos mediante un feedback interactivo

- La distribución de resultados en función del análisis de los datos de entrada recibidos.

Como trabajo futuro y nuevas perspectivas en la evolución del sistema, RedacText 2.0 se plantea la realización de tareas de extensibilidad de la plataforma hacia un sistema seguro, en el que los resultados de la investigación del proyecto sean publicados y se encuentren protegidos bajo usuario y password. Se pretende también desarrollar un módulo que provea a la plataforma de inteligencia para la integración con aplicaciones Web 3.0. Esto posibilitará la segmentación de contenidos para usuarios, en relación con sus objetivos en el proceso de escritura y en relación con su perfil desarrollado en la plataforma. Este perfil debe ser enriquecido con la historia del usuario, a medida que vaya usando RedacText.

Una proyección importante de esta plataforma sería la ampliación de los contenidos del portal para los alumnos de Secundaria y de Primaria, tramos del sistema educativo para los que se forman profesionalmente los actuales usuarios. Dichos tramos pueden ser tanto de modalidad presencial como de educación a distancia. De este modo, podrá obtenerse una amplia gama de resultados en todos los niveles educativos.

\section{Conclusiones}

El testimonio de los estudiantes, de la relatora de la experiencia, y del equipo de investigación permiten constatar que la plataforma RedacText 2.0 favorece el aprendizaje y la evaluación autónomos, a la vez que supone dotar a estos futuros profesionales de una herramienta de interés para transformar sus prácticas de lectura y de escritura en las aulas. La experimentación que se está llevando a cabo deberá refrendar los resultados.

Este trabajo ha planteado multitud de interrogantes. Unos quedan resueltos en el diseño; otros habrán de ser estudiados en la experimentación de la misma.

El principal reto que tiene por delante el Grupo Didactext respecto de esta herramienta consiste en comparar la calidad de los textos producidos sin ayuda y con ayuda de esta plataforma, mediante la evaluación de indicadores textuales de calidad que lo atestigüen.

Son asimismo objeto de interés para el equipo de investigación el estudio de cómo escriben los escritores o estudiantes y cómo enseñan los profesores o tutores con ayuda de la plataforma; qué dicen los escritores y qué hacen y cómo lo hacen, mientras redactan con la plataforma; los comentarios de los tutores y de los escritores: cómo ha funcionado, qué cambios han observado unos y otros, respecto de la concepción de la 
escritura, del planteamiento procesual y de la interacción (actividades y ayudas, principalmente); cómo han funcionado los cuestionarios para ayudar y mediar en la realización de las actividades; cuál es la relación entre los productos de cada fase de escritura y el texto final; cuál es el histórico de cada alumno para analizar los avances y la comparación con otros; cómo han funcionado las redes sociales asociadas a la escritura; etcétera.

Merece destacarse finalmente el hecho de que el diseño de esta plataforma ha supuesto un trabajo conjunto de profesores, estudiantes y técnicos informáticos, debido a la integración de códigos distintos y de visiones complementarias. Son reseñables los esfuerzos realizados por todos, de cara a conseguir el fin propuesto: favorecer la tarea compleja de redactar textos académicos.

\section{Referencias bibliográficas}

ADAM, J. M. (1992). Les textes: types et prototypes. Paris: Nathan.

ÁlVAREZ, T. (2001). Textos expositivo-explicativos y argumentativos. Barcelona: Octaedro.

--- (2005). Didáctica del texto en la formación del profesorado. Madrid: Síntesis.

--- (2006). La escritura de textos expositivos en aulas de primaria. Camps, A. (coord.). (2006a). Diálogo e investigación en las aulas. Investigaciones en didáctica de la lengua, 99-117. Barcelona: Graó.

--- (dir.). (2007). La competencia en comunicación lingüística en las áreas del currículo. Madrid: Ministerio de Educación y Ciencia.

--- (2009). Academic Writing in Spanish Compulsory Education: Improvements after didactic intervention on sixth graders' expository texts. Traditions of Writing Research, 181-197.New York: Routledge.

--- (2010). Competencias básicas en escritura. Barcelona: Octaedro.

--- (2011). Revising and Rewriting in Collaborative Writing in Higher Education and Beyond. Journal of Academic Writing. The Journal of the European Association for the Teaching of Academic Writing (EATAW), 1 (1), 100-109.

--- (2013). Didáctica de la lengua para la formación de maestros. Barcelona: Octaedro.

--- GARCÍA, I. (2011). Presentación: Desarrollo de competencias escritas en los diferentes niveles del sistema educativo. Lenguaje y Textos, Revista de la Sociedad Española de Didáctica de la Lengua y la Literatura, 33, 5-8.

--- RAMÍREZ, R. (2006). Teorías o modelos de producción de textos en la enseñanza y el aprendizaje de la escritura. Didáctica (Lengua y Literatura), 18, 29-60. Madrid: Universidad Complutense.

BAZERMAN, Ch. et al. (2005). Reference Guide to Writing Across the Currículum.Indiana: Pador Press. 
BEAUGRANDE, R., DRESSLER, W. (1972). Introducción a la lingüística del texto, trad. Bonilla, S. (1997). Barcelona: Ariel.

BEREITER, C., SCARDAMALIA, M. (1987). The Psychology of Written Composition. NJ Erlbaum: Hillsdale.

BERNÁRDEZ, E. (comp.). (1987). Lingüística del texto.Madrid: Arco/Libro.

BRITTON, BLACK, J. B. (eds.). (1985). Understanding Expository Text. Hillsdale, New Jersey:LEA.

BRONCKART, J. P. (1996). Activité langagière, textes et discours. Pour un interactionnisme socio discursif. Lausanne-Paris: Delachaux \& Niestlé.

CAMARGO, Z., URIBE, G., CARO, M. A. (2011). Didáctica de la comprensión y producción de textos académicos. ( $2^{\mathrm{a}}$ ed.). Armenia: Universidad del Quindío, Colombia.

CAMPS, A. (comp.). (2003). Secuencias didácticas para aprender a escribir. Barcelona: Graó.

CARLINO, P. (2006). Escribir, leer y aprender en la universidad. Una introducción a la alfabetización Académica. Buenos Aires: Fondo de Cultura Económica.

CASTELLÓ, M. (coord.). (2007). Escribir y comunicarse en contextos científicos y académicos. Conocimientos y estrategias. Barcelona: Graó.

--- DONAHUE, Ch. (eds.). (2012). University writing: selves and texts in academic societies. Bingley: Emerald.

COMBETTES, B. (1988). Pour une grammaire textuelle: la progression thématique. Bruxelles: De Boeck-Wesmal, Paris: J. Duculot.

--- (1992). L'organisation du texte. Université de Metz.

DIDACTEXT, Grupo. (2003). Modelo sociocognitivo, pragmalingüístico y didáctico para la producción de textos escritos. Didáctica (Lengua y Literatura), 15:77-104. Madrid: Universidad Complutense.

--- (2006a). La escritura de textos expositivos en aulas de primaria. Camps, A. (ed.). Diálogo e investigación en las aulas, 99-118. Barcelona, Graó.

--- (2006b). Secuencia didáctica para la escritura de textos expositivos. Textos de Didáctica de la Lengua y de la Literatura, 43: 97-106. Barcelona: Graó.

--- (2007). Los procesos de escritura y el texto expositivo en la mejora de la competencia escrita de los escolares de sexto de educación primaria. Madrid: Universidad Complutense. Línea 300.

DIJK, T. A. van (1997a). El discurso como estructura y proceso. (2000a). Barcelona: Gedisa.

--- (1997b). El discurso como interacción social. (2000b). Barcelona: Gedisa.

DOLZ, J., NOVERRAZ, M., SCHNEUWLY, B. (2001). S' exprimer en français: 
séquences didactiques pour l'oral et pour l'écrit, IV, (13n séquences didactiques 7e-8e-9e). Bruxelles: De Boeck.

DOLZ, J., GAGNON, R., TOULOU, S. (2008). Production écrite et difficultés d'apprentissage. Genève: Universirté. Carnets des sciences de 1 éducation.

ELLISON, N. B. (2007). Social network sites: Definition, history, and scholarship. Journal of Computer-Mediated Communication, 13(1), 210-230.

GARCÍA, I. (coord.). (2011). Escribir textos expositivos en el aula. Fundamentación teórica y secuencias didácticas para diferentes niveles de enseñanza. Barcelona: Graó.

GRABE, W., Kaplan, R. B. (1996). Theory and Practice of Writing. (1998). London: Longman.

GRAVES, D.H. (1983). Didáctica de la escritura. (1991). Madrid: Morata/MEC.

GREGG, L.W., Steinberg. (1980). Cognitive Process in Writing. Hillsdale, NJ: Erlbaum.

HALLIDAY, M.A.K. (1982). Exploraciones sobre las funciones del lenguaje. Barcelona: Médica y Técnica.

--- Martin, J. R. (1993). Writing Science literacy and Discourse Power. London: The Palmer Press.

HALTE, J.F., Petitjean, A., Plane, S. (2002). L'écriture et son apprentissage. Pratiques, 115-116. Metz: Université.

HAMILTON, M., Barton, D. (eds.). (1985). Research and Practice in Adult Literacy. Scheffield: Department of Education Management.

HAYES, J. (1996). Un nuevo marco para la comprensión de lo cognitivo y lo emocional en la escritura. La ciencia de la escritura, 1-27. Madrid: Santillana.

--- Flower, L. (1980). Identifying the organization of writing process. Gregg, L., E. Steinberg (eds.). Cognitive Processes in Writing, 3-30. Hillsdale, N. J.: Lawrence, Erlbaum.

HONEY, C., HERRING, S. C.(2009, January). Beyond microblogging: Conversation and collaboration via Twitter. System Sciences, 1-10. 42nd Hawaii International Conference on IEEE.

HYMESS, D. (1967). Studying the Interaction of Language and Social Life. Foundation in Sociolinguistics. An Ethnographic Approach. London: Tavistock.

JAVA, A., SONG, X., FININ, T., TSENG, B. (2007, August). Why we twitter: understanding microblogging usage and communities. Proceedings of the 9th WebKDD and 1st SNA KDD 2007 workshop on Web mining and social network analysis. ACM., 55-65.

MACARTHUR, CH., GRAHAM, S., J. FITZGERALD (eds.). (2006). Handbook of Writing Research. New York: The Guilford Press. 
NYSTRAND, M. (ed.). (1982). What Writers Know. The Language, Process, and Structure of Written Discourse. New York: Academic Press.

OLIVE, T., LEVY, C. M. (eds.). (2002). Contemporary Tools and Techniques for Studying Writing. The Nederlands: Dordrecht.

OLSON, D.R. (1994). El mundo sobre el papel. El impacto de la escritura y la lectura en la estructura del conocimiento. (1998). Barcelona: Crítica.

RAMÍREZ, R. (2010). Didáctica de la lengua y de la argumentación escrita. Nariño, Colombia: Universidad.

REUTER, Y. (1996). Enseigner et apprendre à écrire. Construire une didactique de l'écriture. Paris: ESF.

ROSE, D., Martin, J. R. (2012). Learning to Write, Reading to Learn: Genre, Knowledge and pedagogy in the Sydney School. London, Equinox.

RUSSEL, D. (2012). Écrits universitaires/écrits professionnalisants /écrits professionnels: est-ce qu'écrire pour apprendre est plus qu'un slogan? Pratiques. $n^{\circ}$ 153-154: 21-34. Université de Metz.

SANMARTI, N. (1996). Para aprender ciencias hace falta aprender a hablar sobre las experiencias y sobre las ideas. Textos de Didáctica de la Lengua y la Literatura, 8: 27-39. Barcelona: Graó.

--- (2007). Hablar, escuchar, leer y escribir para aprender ciencias. Álvarez, T. (coord.). La competencia lingüística en las áreas del currículo, 103-128. Madrid: MEC.

SCHNEUWLY, B. (1988). Le langage écrit chez l'enfant : la production des textes informatifs et argumentatifs. Paris: Delachaux et Niestlé.

SIMARD, Cl., DUFAYS, J.-L, DOLZ, Cl., GARCÍA-DEBANC (2010). Didactique du français langue première. Bruxelles: De Boeck.

VYGOTSKY, L. S. (1932). Pensamiento y lenguaje. (1987). Buenos Aires: La Plèyade.

WASSERMAN, S., FAUST, K. (1994). Social network analysis: Methods and applications, vol. 8. Cambridge: Cambridge University Press.

WRAY, D., Lewis, M. (1997). Aprender a leer y escribir textos de información. (2000). Madrid: Morata. 


\section{Correspondencia con los autores}

Teodoro ÁlVAREZ ANGULO

Facultad de Educación - Universidad Complutense de Madrid

Avda. Rector Royo Villanueva, $\mathrm{s} / \mathrm{n}$

28040 Madrid

e-mail: talang@ucm.es

Teléfono: 34913946166

Miguel Ángel GONZÁLEZ SERRANO

Calle Navarra, 12, Portal $34^{\circ} \mathrm{A}$

28100 Alcobendas

e-mail: miguela.gonzalez@estudiante.uam.es

Teléfono: 665848409

María Teresa MATEO GIRONA

C/ Españoleto, 10, $4^{\circ}$ izq.

28010 Madrid

e-mail: mtmateo@ucm.es

Teléfono: 606263232

$\mathrm{M}^{\mathrm{a}}$ del Pilar SERRANO ALMODÓVAR

Facultad de Educación - Universidad Complutense de Madrid

Despacho 2002,

Avda. Rector Royo Villanueva, $\mathrm{s} / \mathrm{n}$

28040 Madrid

e-mail: seralmo@ucm.es

Teléfono: 913946160 